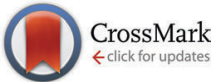

Cite this: Phys. Chem. Chem. Phys., 2016, 18, 17881

Received 5th April 2016

Accepted 7th June 2016

DOI: $10.1039 / c 6 c p 02254 e$

www.rsc.org/pccp

\title{
SANS study on the solvated structure and molecular interactions of a thermo-responsive polymer in a room temperature ionic liquid $\dagger$
}

\author{
Kazu Hirosawa, ${ }^{a}$ Kenta Fujii, ${ }^{b}{ }^{b}$ Takeshi Ueki, ${ }^{c}$ Yuzo Kitazawa, ${ }^{d}$ Kenneth C. Littrell, ${ }^{e}$ \\ Masayoshi Watanabe ${ }^{d}$ and Mitsuhiro Shibayama*a
}

\begin{abstract}
We have utilized small-angle neutron scattering (SANS) to quantitatively characterize the LCST-type phase behavior of the poly(benzyl methacrylate) (PBnMA) derivative poly(2-phenylethyl methacrylate) (PPhEtMA) in the deuterated ionic liquid (IL) $d_{8}$-1-ethyl-3-methylimidazolium bis(trifluoromethanesulfonyl)amide $\left(d_{8}-\left[\mathrm{C}_{2} \mathrm{mlm}^{+}\right]\left[\mathrm{TFSA}^{-}\right]\right)$. The SANS curves showed a discontinuous change from those characteristics of dispersed polymer chains to those of large aggregates of PPhEtMA chains suspended in the IL solution, indicating that phase separation occurs discontinuously at $T_{c}$. Furthermore, we evaluated the enthalpic and entropic contributions to the effective interaction parameter $\chi_{\text {eff }}$ of PPhEtMA in $\left[\mathrm{C}_{2} \mathrm{mlm}^{+}\right]\left[T F S A^{-}\right]$and compared them with those of PBnMA. The absolute value of the enthalpic contribution observed for PPhEtMA was smaller than that for PBnMA. This difference in the enthalpic term can be attributed to the unfavorable interaction between the $\mathrm{IL}$ and the alkyl group in the side chain of PPhEtMA. In addition, the temperature dependence of $\chi_{\text {eff }}$ was smaller than the previously reported value for a thermo-responsive polymer in an aqueous system. It was found out that the strong dependence of $T_{\mathrm{C}}$ on the chemical structure in $\mathrm{IL}$ systems originated from the relatively smaller temperature dependence of $\chi_{\text {eff }}$.
\end{abstract}

\section{Introduction}

Ionic liquids (ILs) consist of only ion species. Thus, they exhibit unique solvent properties such as high ion conductivity, electrochemical stability, negligible volatility and nonflammability. ${ }^{3}$ More importantly, the solvent properties of ILs can be tuned by selecting appropriate combinations of ions, and they can dissolve various solutes including metal ions, ${ }^{4,5}$ organic molecules ${ }^{6,7}$ and biopolymers. ${ }^{8-11}$ In polymer science, the combination of polymer materials with appropriate ILs permits the fabrication of functional soft materials by exploiting the unique properties of ILs. ${ }^{12-17}$ Recently, various systems of different types of stimuli-responsive

${ }^{a}$ Institute for Solid State Physics, The University of Tokyo, 5-1-5 Kashiwanoha, Kashiwa, Chiba 277-8581, Japan. E-mail: sibayama@issp.u-tokyo.ac.jp

${ }^{b}$ Graduate School of Science and Engineering, Yamaguchi University,

2-16-1 Tokiwadai, Ube, Yamaguchi 755-8611, Japan.

E-mail:k-fujii@yamaguchi-u.ac.jp

${ }^{c}$ Polymer Materials Unit, National Institute for Materials Science,

1-1 Namiki, Tsukuba, Ibaraki, 305-0044, Japan

${ }^{d}$ Department of Chemistry and Biotechnology, Yokohama National University,

79-5 Tokiwadai, Hodogaya-ku, Yokohama 240-8501, Japan

${ }^{e}$ Oak Ridge National Laboratory, 1 Bethel Valley Road, Oak Ridge, Tennessee, 37831 USA

$\dagger$ Electronic supplementary information (ESI) available: Mass density of the material, viscosity of PBnMA in $\left[\mathrm{C}_{2} \mathrm{mIm}^{+}\right]\left[\mathrm{TFSA}^{-}\right]$solutions and $\chi_{\text {eff }}$ calculated from $A_{2}$. See DOI: $10.1039 /$ c6cp02254e

polymers in ILs have been developed for applications to sensors, actuators and switching devices possessing the intrinsic properties of the ILs. ${ }^{1,18-27}$ As it is well-known, poly( $N$-isopropylacrylamide) (PNIPAm) shows a lower critical solution temperature (LCST)-type phase separation in aqueous solutions. In contrast, PNIPAm exhibits an upper critical solution temperature (UCST)-type phase separation in a typical hydrophobic IL, 1-ethyl-3-methylimidazolium bis(trifluoromethanesulfonyl)amide $\left(\left[\mathrm{C}_{2} \mathrm{mIm}^{+}\right]\left[\mathrm{TFSA}^{-}\right]\right.$, as shown in Scheme 1). ${ }^{18}$ Interestingly, it was found for the first time that poly(benzyl methacrylate) (PBnMA, shown in Scheme 1)

(a) PBnMA<smiles>CCC(C)(CC)C(=O)OCc1ccccc1</smiles>

(c) $\left[\mathrm{C}_{2} \mathrm{mIm}^{+}\right]\left[\mathrm{TFSA}^{-}\right]$

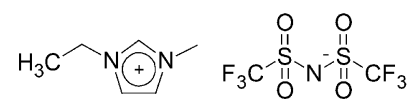

Scheme 1 Schematic illustration for the chemical structure of (a) PPhEtMA, (b) PBnMA and (c) $\left[\mathrm{C}_{2} \mathrm{mlm}^{+}\right]\left[T F S A^{-}\right]$, together with the reported phase separation temperature, $T_{\mathrm{c}}$ 's of PPhEtMA and PBnMA in $\left[\mathrm{C}_{2} \mathrm{mlm}^{+}\right]\left[T F S A^{-}\right]^{1}$ 
and its derivatives show an LCST-type phase separation in $\left[\mathrm{C}_{2} \mathrm{mIm}^{+}\right]\left[\mathrm{TFSA}^{-}\right] .^{1,19}$ Furthermore, a cross-linked PBnMA gel in $\left[\mathrm{C}_{2} \mathrm{mIm}^{+}\right]\left[\mathrm{TFSA}^{-}\right]$shows discontinuous LCST-type volume phase transition in response to the temperature change. ${ }^{19}$ One of the characteristics of stimuli-responsive polymers in IL systems is that their phase transition temperature $\left(T_{\mathrm{c}}\right)$ strongly depends on both the polymer chemical structures and the structures of the ILs. For example, the LCST-type phase transition temperature of poly(2-phenylethyl methacrylate) (PPhEtMA, shown in Scheme 1) is $63 \mathrm{~K}$ lower than that of PBnMA although the chemical structures of PPhEtMA and PBnMA differ only by one methylene spacer inserted in the side chains. ${ }^{1}$ This strong dependence of $T_{c}$ on the chemical structure provides a mechanism by which one can control the phase behavior of polymers in IL systems by designing the chemical structure of polymers or ILs appropriately.

In a previous study, we studied PBnMA in the $\left[\mathrm{C}_{2} \mathrm{mIm}^{+}\right]\left[\mathrm{TFSA}^{-}\right]$ system using small-angle neutron scattering (SANS). In this system, it was found that the PBnMA chains exist as independent Gaussian chains in $\left[\mathrm{C}_{2} \mathrm{mIm}^{+}\right]\left[\mathrm{TFSA}^{-}\right]$below $T_{\mathrm{c}}$ but suddenly form aggregates at $T_{\mathrm{c}}{ }^{2}$ Furthermore, our investigations of the microscopic solvation structure of PBnMA in $\left[\mathrm{C}_{2} \mathrm{mIm}^{+}\right]\left[\mathrm{TFSA}^{-}\right]$by high-energy X-ray total scattering (HEXTS) modeled with the aid of MD simulations demonstrated that the $\mathrm{C}_{2} \mathrm{mIm}^{+}$cations are localized above and below the benzyl groups of PBnMA. ${ }^{28}$ This partially ordered microscopic solvation structure of PBnMA in $\left[\mathrm{C}_{2} \mathrm{mIm}^{+}\right]\left[\mathrm{TFSA}^{-}\right]$solution decreases the mixing entropy, resulting in the observed LCSTtype phase behavior. From a thermodynamic aspect, it was reported that the mixing enthalpy and entropy values of PBnMA in $\left[\mathrm{C}_{2} \mathrm{mIm}^{+}\right]\left[\mathrm{TFSA}^{-}\right]$are significantly smaller than those of PNIPAm in $\mathrm{H}_{2} \mathrm{O}$, indicating that $T_{\mathrm{c}}$ of the system can be easily modified by a slight change in the free energy of mixing. ${ }^{29}$ Although several research groups have studied the structural aspects of thermo-responsive polymers in IL systems using infrared spectroscopy, ${ }^{30}$ SANS, ${ }^{31-33}$ and theoretical investigations of the phase diagram, ${ }^{34}$ the correlation between the microscopic or the mesoscopic solvation structure and macroscopic phase behavior is still unclear. For example, the physical origin of the above-mentioned differences in the $T_{\text {c }}$ 's of PBnMA and PPhEtMA is completely unknown.

In this study, we chose PPhEtMA in $\left[\mathrm{C}_{2} \mathrm{mIm}^{+}\right]\left[\mathrm{TFSA}^{-}\right]$ solution as a model system for investigating the key factors that determine the phase behavior of polymers in IL systems. We performed SANS measurements on this system and carried out quantitative data analysis to determine the thermodynamic parameters such as the second virial coefficient, $A_{2}$, the effective interaction parameter, $\chi_{\text {eff }}$, and the $\Theta$ temperature. The enthalpic and entropic contributions to $\chi_{\text {eff }}$ were estimated from temperature dependence of $\chi_{\text {eff }}$ and compared with those obtained for PBnMA.

This study is an attempt to understand the phase behavior of polymers in IL systems from a viewpoint of microscopic molecular interactions. Such physicochemical characterization of the phase behavior will contribute enormously to facilitating a systematic design of novel systems of polymers in ILs.

\section{Experimental}

\section{Materials}

PPhEtMA was prepared by atom transfer radical polymerization (ATRP) following a previously-reported procedure. ${ }^{35} \mathrm{CuBr} 5.8 \mathrm{mg}$ and $\mathrm{CuBr}_{2} 0.9 \mathrm{mg}$ were dried at $60{ }^{\circ} \mathrm{C}$ in a $50 \mathrm{~mL}$ Schlenk flask under vacuum and then stored in a nitrogen atmosphere. Anisole $10 \mathrm{~mL}$ and $N, N, N^{\prime}, N^{\prime \prime}, N^{\prime \prime}$-pentamethyldiethylenetriamine (PMDETA) $10.3 \mu \mathrm{L}$ were added to the flask and stirred until the mixture became transparent. 2-Phenylethyl methacrylate (PhEtMA) $10 \mathrm{~mL}$ and the initiator (2-bromoisobutyric acid ethyl ester) $23.9 \mu \mathrm{L}$ were added and the system was degassed twice using freeze-pump-thaw cycles. The polymerization reaction of PhEtMA was conducted under a nitrogen atmosphere at $40{ }^{\circ} \mathrm{C}$ for $6 \mathrm{~h}$, before termination by quenching with dry ice and methanol. The resulting product was purified by reprecipitation three times using ethyl acetate as the good solvent and methanol as the poor solvent and dried for $24 \mathrm{~h}$ at room temperature under vacuum. The structure of the polymer was characterized using ${ }^{1} \mathrm{H}-\mathrm{NMR}$ spectroscopy and size exclusion chromatography (SEC). SEC was conducted using $N, N$-dimethylformamide (DMF) containing $0.01 \mathrm{~mol} \mathrm{~L}^{-1} \mathrm{LiBr}$ as the eluent. The number average molecular weight, $M_{\mathrm{n}}$, was determined to be $M_{\mathrm{n}}=31.5 \mathrm{k}$ by ${ }^{1} \mathrm{H}-\mathrm{NMR}$ and the polydispersity index $\left(M_{\mathrm{w}} / M_{\mathrm{n}}\right)$ was measured to be $M_{\mathrm{w}} / M_{\mathrm{n}}=1.30$ using Tosoh columns calibrated with PMMA standards, where $M_{\mathrm{w}}$ is the weight average molecular weight.

The ionic liquid $\left[\mathrm{C}_{2} \mathrm{mIm}^{+}\right]\left[\mathrm{TFSA}^{-}\right]$and the partially deuterated ionic liquid $d_{8}-\left[\mathrm{C}_{2} \mathrm{mIm}^{+}\right]\left[\mathrm{TFSA}^{-}\right]$whose $\mathrm{H}$ atoms are substituted by $\mathrm{D}$ for the ethyl group and the imidazolium-ring of the $\mathrm{C}_{2} \mathrm{mIm}$ cation but not for the methyl group were synthesized according to the procedures reported before. ${ }^{36,37}$ The deuteration ratio of $d_{8}-\left[\mathrm{C}_{2} \mathrm{mim}^{+}\right]\left[\mathrm{TFSA}^{-}\right]$was estimated to be higher than $93 \%$ based on ${ }^{1} \mathrm{H}$-NMR spectroscopy measurements. All of the sample solutions were prepared using the cosolvent evaporation method with THF as the good solvent. ${ }^{38,39}$

\section{Viscosity measurements}

Viscosity measurements were carried out on PPhEtMA in $\left[\mathrm{C}_{2} \mathrm{mIm}^{+}\right]$$\left[\mathrm{TFSA}^{-}\right]$solutions using a rheometer (MCR501, Anton Paar, Austria) at $298 \mathrm{~K}$ with a cone-plate geometry and a shear rate of $100 \mathrm{~s}^{-1}$.

\section{Dynamic light scattering (DLS) measurements}

DLS measurements were carried out using a SLS/DLS 5022F-PCCMS compact goniometer (ALV, Langen) coupled with an ALV photon correlator. A $22 \mathrm{~mW} \mathrm{He}-\mathrm{Ne}$ laser (wavelength, $\lambda=632.8 \mathrm{~nm}$ ) was used as the incident beam and the scattering angle $\theta$ was $90^{\circ}$. Each measurement required $30 \mathrm{~s}$. The solvent IL $\left[\mathrm{C}_{2} \mathrm{mIm}^{+}\right]\left[\mathrm{TFSA}^{-}\right]$was passed through a PTFE filter (pore size: $0.5 \mu \mathrm{m}$ ) prior to use. The temperature was changed at a rate of approximately $0.5 \mathrm{~K} \mathrm{~min}^{-1}$, and DLS measurements were performed at each temperature after allowing the system to stabilize for at least $5 \mathrm{~min}$.

The distribution function of the hydrodynamic radius $R_{\mathrm{h}}$ was obtained by applying the inverse Laplace transformation to the time correlation function of scattering intensity using the well-established CONTIN program. ${ }^{40}$ The temperature dependence of the viscosity of $\left[\mathrm{C}_{2} \mathrm{mIm}^{+}\right]\left[\mathrm{TFSA}^{-}\right]$was determined using the 
Vogel-Tammann-Fulcher (VTF) equation as previously reported. ${ }^{41}$ The refractive index of $\left[\mathrm{C}_{2} \mathrm{mIm}^{+}\right]\left[\mathrm{TFSA}^{-}\right]$at each temperature was obtained from a previous report. ${ }^{42}$

\section{Cloud point observation}

PPhEtMA in $\left[\mathrm{C}_{2} \mathrm{mIm}^{+}\right]\left[\mathrm{TFSA}^{-}\right]$and $d_{8^{-}}\left[\mathrm{C}_{2} \mathrm{mIm}^{+}\right]\left[\mathrm{TFSA}^{-}\right]$solutions was poured into test tubes immersed in a water bath. Temperature was elevated at a rate slower than $0.2 \mathrm{~K} \mathrm{~min}^{-1}$, and the cloud point was determined by visual observation. We measured the highest temperature at which sample solution looked completely transparent $\left(T_{1}\right)$ and the lowest one at which it looked completely opaque $\left(T_{2}\right)$. We defined the cloud point $\left(T_{\mathrm{c}}\right)$ as the middle between $T_{1}$ and $T_{2}$, i.e., $T_{\mathrm{c}}=\left(T_{1}+T_{2}\right) / 2$, and defined the uncertainty of $T_{\mathrm{c}}\left(\Delta T_{\mathrm{c}}\right)$ as $\Delta T_{\mathrm{c}}=\left(T_{2}-T_{1}\right) / 2$.

\section{SANS measurements}

PPhEtMA in $d_{8}-\left[\mathrm{C}_{2} \mathrm{mIm}^{+}\right]\left[\mathrm{TFSA}^{-}\right]$solutions of polymer concentration, $c=8-29 \mathrm{mg} \mathrm{mL} \mathrm{mL}^{-1}$ were injected into $2 \mathrm{~mm}$ thick spectroscopic grade quartz "banjo" cells, each containing $700 \mu \mathrm{l}$ of the sample. The temperature was changed from $288 \mathrm{~K}$ to $310 \mathrm{~K}$ at a rate of $0.5 \mathrm{~K} \mathrm{~min}^{-1}$; the SANS measurements were performed at each temperature after the solution had stabilized for at least $20 \mathrm{~min}$. The SANS measurements were performed at the CG-2 General Purpose SANS instrument in the High Flux Isotope Reactor (HFIR) facility in Oak Ridge National Laboratory (ORNL, USA). Two instrument configurations were used to yield a $q$-range from $0.0028 \AA^{-1}-0.46 \AA^{-1}$ where $q$ is the magnitude of the scattering vector defined as $q=(4 \pi / \lambda) \sin \theta$ and $2 \theta$ is the scattering angle. The configurations were (i) source-to-sample distance $(\mathrm{SSD})=17.3 \mathrm{~m}$, sample-to-detector distance $(\mathrm{SDD})=19.3 \mathrm{~m}$ and (ii) $\mathrm{SSD}=5.1 \mathrm{~m}$ and $\mathrm{SDD}=2.8 \mathrm{~m}$. Both configurations used a wavelength of $\lambda=4.72 \AA$.

The measured data were corrected for background scattering (empty sample holder), transmission and detector efficiency according to the standard protocol using the Igor Pro based software provided by ORNL. The corrected scattering profiles were scaled to absolute units using a calibrated attenuated direct-beam measurement. The coherent scattering intensity from the sample solutions was obtained by the subtraction of the incoherent scattering calculated from chemical composition. We subtracted the coherent scattering intensity of the neat $d_{8^{-}}\left[\mathrm{C}_{2} \mathrm{mIm}^{+}\right]\left[\mathrm{TFSA}^{-}\right]$from those of sample solutions to obtain the coherent scattering intensity of PPhEtMA in $d_{8}-\left[\mathrm{C}_{2} \mathrm{mIm}^{+}\right]\left[\mathrm{TFSA}^{-}\right]$, referred to hereafter as $I(q)$.

The mass densities of $\left[\mathrm{C}_{2} \mathrm{mIm}^{+}\right]\left[\mathrm{TFSA}^{-}\right], d_{8}\left[\mathrm{C}_{2} \mathrm{mIm}^{+}\right]\left[\mathrm{TFSA}^{-}\right]$ and PhEtMA (monomer) were measured at $298 \mathrm{~K}$ using a digital density meter (DMA 4500, Anton Paar, Austria) to provide the values used for analysis; the results of density measurements are summarized in Table S1 (ESI $\dagger$ ).

\section{Results and discussion}

\section{Cloud points of PPhEtMA in $\left[\mathrm{C}_{2} \mathrm{mIm}^{+}\right]\left[\mathrm{TFSA}^{-}\right]$and $d_{8}-\left[\mathrm{C}_{2}\right.$ mIm $\left.^{+}\right]\left[\right.$TFSA $\left.^{-}\right]$}

We first determined the cloud points $T_{\mathrm{c}}$ of PPhEtMA in $\left[\mathrm{C}_{2} \mathrm{mIm}^{+}\right]\left[\mathrm{TFSA}^{-}\right]$and $d_{8^{-}}\left[\mathrm{C}_{2} \mathrm{mIm}^{+}\right]\left[\mathrm{TFSA}^{-}\right]$solutions by visual

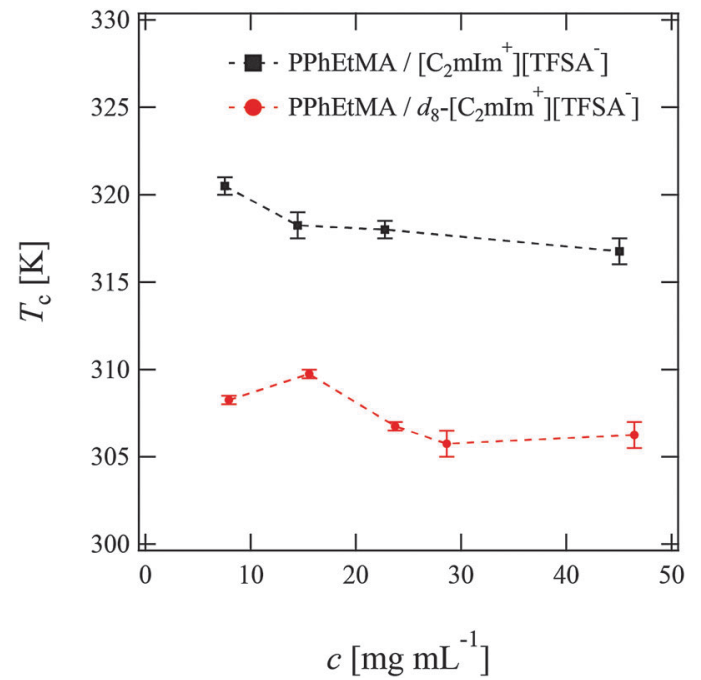

Fig. 1 The cloud points of PPhEtMA in $\left[C_{2} \mathrm{mlm}^{+}\right]\left[\mathrm{TFSA}^{-}\right]$(black squares) and $d_{8}-\left[\mathrm{C}_{2} \mathrm{mlm}^{+}\right]\left[\mathrm{TFSA}^{-}\right.$] (red circles) solution at various concentration as obtained by visual observation. The broken lines are guides to eye. The error bars represent the uncertainty of $T_{c}, \Delta T_{c}$.

observation. Fig. 1 shows the concentration dependence of $T_{\mathrm{c}}$. As Fig. 1 indicates, the $T_{\mathrm{c}}$ of PPhEtMA was in the range of $306 \mathrm{~K}-310 \mathrm{~K}$ in $d_{8^{-}}\left[\mathrm{C}_{2} \mathrm{mIm}^{+}\right]\left[\mathrm{TFSA}^{-}\right]$which is significantly lower than in protonated $\left[\mathrm{C}_{2} \mathrm{mIm}^{+}\right]\left[\mathrm{TFSA}^{-}\right](315-320 \mathrm{~K})$. Such a difference of $T_{\mathrm{c}}$ in deuterated and protonated solvents has been previously reported for PNIPAm in aqueous solutions, ${ }^{43-45}$ poly(styrene) in cyclohexane solutions, ${ }^{46}$ polymers in IL solutions, ${ }^{31}$ and for polymer blends. ${ }^{47,48}$ For example, the $T_{\mathrm{c}}$ of PNIPAm in $\mathrm{D}_{2} \mathrm{O}$ was reported to be $0.6-0.8 \mathrm{~K}$ higher than in $\mathrm{H}_{2} \mathrm{O}$, due to the more stable hydrogen-bonding interactions in $\mathrm{D}_{2} \mathrm{O}$.

The effect of deuteration on $T_{\mathrm{c}}$ originates mainly from differences in molecular interactions in deuterated and protonated solvents. ${ }^{31,44,45}$ It is thus expected that the observed differences in $T_{\mathrm{c}}$ of PPhEtMA in protonated and deuterated ILs similarly originate from the isotope effect on the strength of molecular interactions. The molar volume of neat $d_{8}-\left[\mathrm{C}_{2} \mathrm{mIm}^{+}\right]\left[\mathrm{TFSA}^{-}\right]$ $\left(2.5730 \times 10^{-4} \mathrm{~m}^{3} \mathrm{~mol}^{-1}\right)$ calculated from its mass density (see Table S1, ESI $\dagger$ ) was slightly smaller than that of the protonated $\left[\mathrm{C}_{2} \mathrm{mIm}^{+}\right]\left[\mathrm{TFSA}^{-}\right]\left(2.5777 \times 10^{-4} \mathrm{~m}^{3} \mathrm{~mol}^{-1}\right)$. This result indicates that cohesive interactions between IL ions are slightly enhanced by deuterium substitution. As mentioned in the Introduction, it has been reported previously that the enthalpy and entropy of mixing of PBnMA in $\left[\mathrm{C}_{2} \mathrm{mIm}^{+}\right]\left[\mathrm{TFSA}^{-}\right]$are quite small compared to those of PNIPAm in the $\mathrm{H}_{2} \mathrm{O}$ system. ${ }^{29}$ Hence, even a slight difference in the interactions can affect the $T_{\mathrm{c}}$ of PPhEtMA, one of the PBnMA derivatives in the IL. We conclude that the lower $T_{\mathrm{c}}$ of PPhEtMA in $d_{8}-\left[\mathrm{C}_{2} \mathrm{mIm}^{+}\right]\left[\mathrm{TFSA}^{-}\right]$and the larger difference in $T_{\mathrm{c}}$ 's $(\approx 10 \mathrm{~K})$ can be ascribed to the slightly stronger cohesive interactions between deuterated IL ions compared to those between protonated ones.

However, it should be noted that $T_{\mathrm{c}}$ decreases for PPhEtMA in an IL system while it increases for PNIPAm in water by deuterium substitution on solvents. This opposite tendency suggests that the solvation mechanism is different between ILs 
and aqueous systems. Here, the former is ascribed to the sidechain "philic" interaction between the phenyl group of PPhEtMA and the $\mathrm{C}_{2} \mathrm{mIm}$ cation, ${ }^{28}$ while the latter in the side-chain "phobic" interaction is stabilized by the solvent-solvent interaction. ${ }^{44,45,49}$ When the cohesive interaction between solvent molecules is enhanced by deuterium substitution, ${ }^{46}$ the side-chain "philic" interaction should become unfavorable whereas the sidechain "phobic" interaction favorable. We thus conjecture that the opposite deuteration effects on $T_{\mathrm{c}}$ of polymers in ILs and in water solutions reflect the difference in their solvation, i.e., the side-chain "philic" interaction of PPhEtMA in the $\left[\mathrm{C}_{2} \mathrm{mIm}^{+}\right]\left[\mathrm{TFSA}^{-}\right]$system and the side-chain "phobic" interaction of PNIPAm in the water system.

\section{Viscosity measurements}

Fig. 2 shows the reduced viscosity $\eta_{\text {red }}$ of PPhEtMA in $\left[\mathrm{C}_{2} \mathrm{mIm}^{+}\right]\left[\mathrm{TFSA}^{-}\right]$solutions as a function of PPhEtMA concentration $c$, where $\eta_{\text {red }}$ is defined by the following equation:

$$
\eta_{\mathrm{red}}=\frac{\eta-\eta_{0}}{c \eta_{0}}
$$

Here, $\eta$ and $\eta_{0}$ are the viscosities of the solution and the solvent, respectively. The chain-overlapping concentration $\left(c^{*}\right)$ was determined by the following equation;

$$
c^{*}=\frac{3 \times 6^{3 / 2} \Phi}{4 \pi N_{\mathrm{A}}[\eta]}
$$

where $\Phi$ is the universal constant. We employed the value of $\Phi=2.1 \times 10^{23} \mathrm{~mol}^{-1}$ in our analysis. ${ }^{50,51}$ The intrinsic viscosities, $[\eta]$, were obtained by extrapolating $\eta_{\text {red }}$ to $c \rightarrow 0$, as shown by the solid line in Fig. 2. The $c^{*}$ value was estimated to be approximately $130 \mathrm{mg} \mathrm{mL} \mathrm{m}^{-1}$; we performed the DLS and SANS measurements for PPhEtMA in $\left[\mathrm{C}_{2} \mathrm{mIm}^{+}\right]\left[\mathrm{TFSA}^{-}\right]$solution systems at concentrations lower than the estimated $c^{*}$.



Fig. 2 Concentration dependence of the reduced viscosities $\left(\eta_{\text {red }}\right)$ of PPhEtMA in $\left[\mathrm{C}_{2} \mathrm{mlm}^{+}\right]\left[\mathrm{TFSA}^{-}\right]$solutions. The intrinsic viscosities $([\eta])$ were obtained by extrapolating $\eta_{\text {red }}$ to $c=0$ (solid line).

\section{DLS measurements}

Fig. 3(a) and (b) show the time-correlation function $g^{(2)}(\tau)-1$ and the corresponding $R_{\mathrm{h}}$ distribution functions $G\left(R_{\mathrm{h}}\right)$ observed for $15 \mathrm{mg} \mathrm{mL} \mathrm{m}^{-1}$ PPhEtMA solution at various temperatures, respectively. The DLS measurements were carried out below the phase-separating temperature as the solution became opaque and strong multiple scattering prevented DLS measurements above the phase-separating temperature. The $G\left(R_{\mathrm{h}}\right)$ s data at $T<303 \mathrm{~K}$ showed a single intense peak at around $R_{\mathrm{h}} \sim 45-50 \AA$ with the peak positions independent of temperature. Above $303 \mathrm{~K}$, a small peak (slow-mode peak) appeared at $R_{\mathrm{h}}>1000 \AA$ while the peak at $R_{\mathrm{h}} \sim 45-50 \AA$ remained intense. This slow-mode peak was also observed in the previous study of PBnMA in the $\left[\mathrm{C}_{2} \mathrm{mIm}^{+}\right]\left[\mathrm{TFSA}^{-}\right]$system; it indicates the appearance of local micro-phase separation below the bulk phase separation temperature. ${ }^{2}$ This result suggests that the majority of the PPhEtMA chains are homogeneously dispersed but that a small amount of aggregates coexists at the temperature near the critical temperature.

Fig. 4 shows the temperature dependence of $R_{\mathrm{h}}$ of PPhEtMA chains corresponding to the fast mode. It is clear that $R_{\mathrm{h}}$ was almost independent of temperature, indicating that molecular conformation of solvated PPhEtMA chains remained essentially unchanged across the examined temperature range.

\section{SANS measurements}

We performed SANS measurements for the present system using deuterated IL, $d_{8}-\left[\mathrm{C}_{2} \mathrm{mIm}^{+}\right]\left[\mathrm{TFSA}^{-}\right]$, as the solvent to investigate the chain conformation and evaluate thermodynamic parameters for PPhEtMA in the $\left[\mathrm{C}_{2} \mathrm{mIm}^{+}\right]\left[\mathrm{TFSA}^{-}\right]$system. The SANS curves observed for PPhEtMA in $d_{8^{-}}\left[\mathrm{C}_{2} \mathrm{mIm}^{+}\right]\left[\mathrm{TFSA}^{-}\right]$solution $\left(c=16 \mathrm{mg} \mathrm{mL}^{-1}\right)$ at various temperatures are shown in Fig. 5 . Here, $c$ of the examined sample solution was substantially lower than $c^{*}\left(\sim 130 \mathrm{mg} \mathrm{mL}^{-1}\right)$ and the system was in the dilute region. As Fig. 5 indicates, the scattering intensity slightly increased with increasing temperature, but then showed a steep
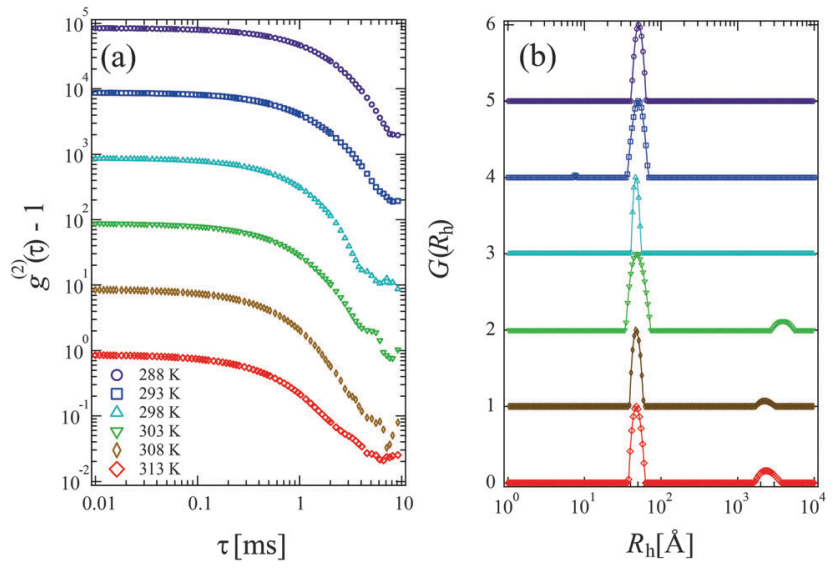

Fig. 3 (a) The time correlation functions of scattering intensity, $g^{(2)}(\tau)-1$ and (b) the distribution functions of the hydrodynamic radius, $G\left(R_{\mathrm{h}}\right)$ obtained for PPhEtMA in $\left[\mathrm{C}_{2} \mathrm{mlm}^{+}\right]\left[\mathrm{TFSA}^{-}\right]$solution of $c=15 \mathrm{mg} \mathrm{mL}^{-1}$ at various temperatures. The correlation functions in (a) are vertically shifted for visibility. The solid lines in (b) are guide to eye. 


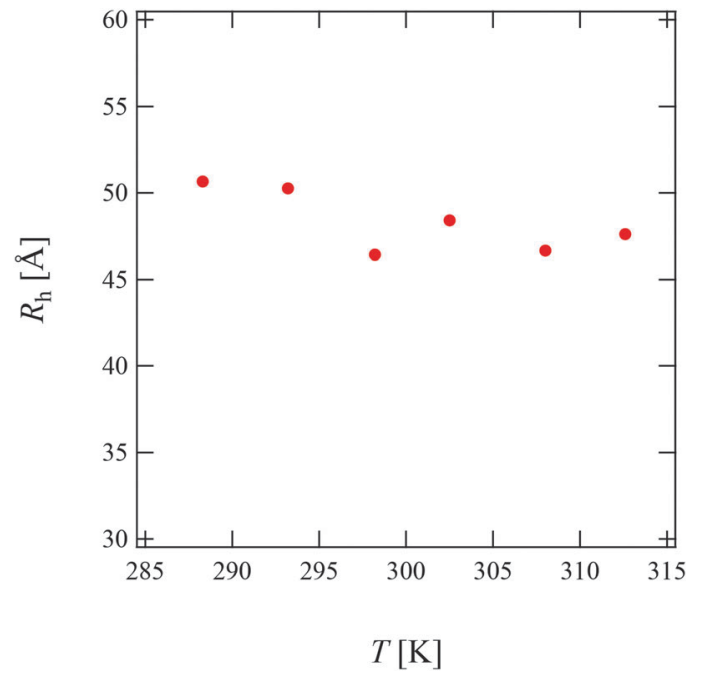

Fig. 4 The temperature dependence of the $R_{\mathrm{h}}$ values obtained for the PPhEtMA in $\left[\mathrm{C}_{2} \mathrm{mlm}^{+}\right]\left[T F S A^{-}\right]$solution at $c=15 \mathrm{mg} \mathrm{mL}^{-1}$.

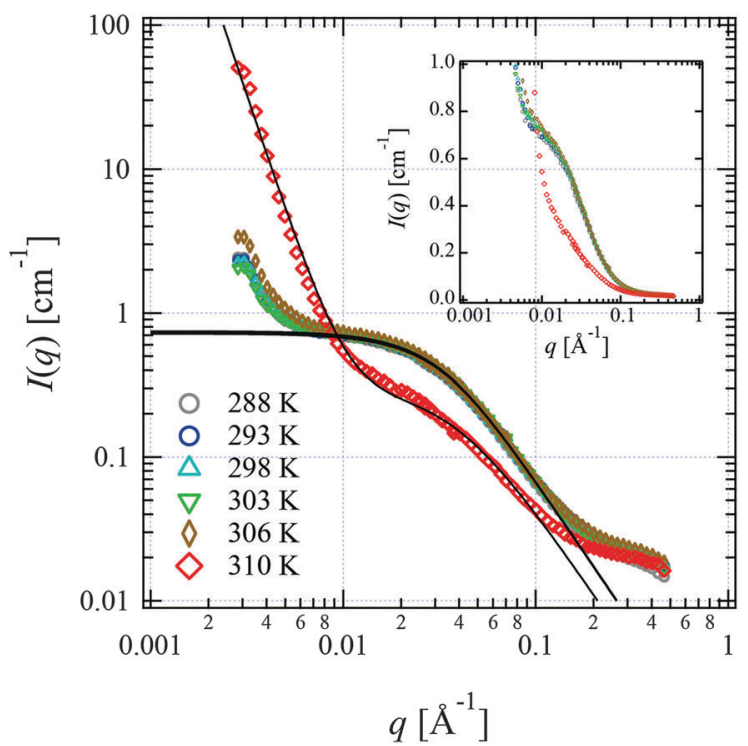

Fig. 5 SANS profiles observed for the PPhEtMA in $d_{8}-\left[\mathrm{C}_{2} \mathrm{mlm}^{+}\right]\left[\mathrm{TFSA}^{-}\right]$ solution ( $c=16 \mathrm{mg} \mathrm{mL}^{-1}$ ) at various temperatures shown in the form of the $\log -\log$ plot. The solid lines are the fits with eqn (3) or eqn (3) $+\mathrm{Aq}^{-4}$ functions. The SANS profiles are also shown in the form of the semi-log plot in the inset.

upturn in the data at temperatures higher than $T_{\mathrm{c}}(\sim 309 \mathrm{~K}$, see Fig. 1). Here, the upturn at the low- $q$ region $\left(q<0.006 \AA^{-1}\right)$ and the slope in the middle- $q$ region $\left(0.03 \AA^{-1}<q<0.13 \AA^{-1}\right)$ are described by $I(q) \sim q^{-4}$ and $I(q) \sim q^{-2}$, respectively. We modeled the data by fitting to the following scattering function based on Zimm-approximation; ${ }^{52-54}$

$$
\begin{aligned}
I(q) & =\frac{(\Delta \rho)^{2}}{N_{\mathrm{A}}} \frac{V_{2} \phi P_{\mathrm{D}}(q)}{1+\left(1-2 \chi_{\mathrm{eff}}\right)\left(\frac{V_{2}}{V_{1}}\right) \phi P_{\mathrm{D}}(q)} \\
P_{\mathrm{D}}(q) & =\frac{2}{x^{2}}[\exp (-x)-1+x]\left(x \equiv R_{\mathrm{g}}{ }^{2} q^{2}\right)
\end{aligned}
$$

where, $\varphi, \chi_{\text {eff }}, V_{1}$ and $V_{2}$ are the volume fraction of PPhEtMA, the effective interaction parameter, and the molar volumes of the solvent and solute components, respectively. Here, $N_{\mathrm{A}}$ is the Avogadro number, $R_{\mathrm{g}}$ is the radius of gyration, and $\Delta \rho$ is the difference in the scattering length density between the solute and the solvent. This function is well-established as the scattering function describing Gaussian chains in a dilute solution where a single-contact intermolecular interference is taken into account. The solid lines in Fig. 5 are the results of fitting the data using the functions described in eqn (3). As shown here, the scattering profiles below the critical temperature are well reproduced by eqn (3) except for a deviation from the theoretical curve in the low- $q$ region of $q<0.006 \AA^{-1}$ and in the high- $q$ region of $q>0.2 \AA^{-1}$. Here, $R_{\mathrm{g}}$ and $\chi_{\text {eff }}$ of dispersed PPhEtMA chains were obtained from curve fitting. The obtained $R_{\mathrm{g}}$ was almost constant below $T_{\mathrm{c}}$ (Fig. S3, ESI $\dagger$ ). This result indicates that the conformation of PPhMA chains does not change below $T_{\mathrm{c}}$. We will discuss $\chi_{\text {eff }}$ in detail in the later section. The slight upturn in the low- $q$ region is attributed to the existence of small amounts of cluster observed in DLS as the slow mode in DLS (see Fig. 3) and is often observed for polymer solutions. ${ }^{55,56}$ We can neglect this upturn in the curve fitting because the fraction of such a cluster in the system should be small and considering the fact that scattering intensity from them was very weak. In the high- $q$ region, the absolute value of the exponent of $I(q)$ against $q$ was smaller than 2 , indicating the deviation from the Gaussian conformation on the microscopic scale. In previous studies on aqueous solution of hydrophobic polyelectrolytes, ${ }^{57,58}$ it is reported that the collapsed region ("pearl") and the elongated region ("necklace") coexist in a single chain. This structure is called the "pearl-necklace structure". In the present study, as mentioned above, the exponent of $I(q) v s . q$ showed a deviation from -2 at around $q^{*}=0.2 \AA$. We conjecture that such a deviation reflects the existence of locally rigid structures consisting of PPhEtMA chains and their aggregates, i.e., the "pearlnecklace structure". We roughly estimated the characteristic length scale, $R^{*}$ of "pearl" from $q^{*}$, as $R^{*} \sim 10 \AA$. Here, we used the relationship of $R^{*}=1.91 q^{*-1}$ which is often used to estimate the persistence length of these wormlike chains. ${ }^{59}$ We will not dwell further on this point as our primary concern in this study is determining the mesoscopic structure of the system and the phase separation process.

For the scattering profiles at $T>309 \mathrm{~K}$, we performed curve fitting by using a sum of eqn (3) and Debye-Bueche functions,

$$
I(q)=\frac{I(0)}{\left(1+\Xi^{2} q^{2}\right)^{2}} \approx \frac{I(0)}{q^{4}} \quad(q \gg \Xi)
$$

where $\Xi$ is the characteristic size of inhomogeneities in the phase separated structure. In this study, we employed $I(q)=$ eqn (3) $+A q^{-4}$ as a first approximation because of the absence of experimental data in the low- $q$ region. Eventually, the scattering profiles at $T=310 \mathrm{~K}$ were successfully reproduced by eqn (3) with the Debye-Bueche term. It was impossible to estimate the size of aggregates only from SANS profiles because of the lack of data in the low- $q$ region. In the DLS results (see Fig. 3), a slow-mode peak was observed near the critical temperature which corresponds to the size of a small amount of aggregates, $R_{\mathrm{h}}>1000 \AA$. Based on these results, we presume 
that the size of the aggregates formed above the critical temperature is at least $1000 \AA$. These results show that PPhEtMA chains are homogeneously dispersed as the Gaussian chains before the phase separation, and that they suddenly form large aggregates at the critical temperature.

Most previous studies in the literature were performed using semi-dilute solution of phase-separating polymers in water, ${ }^{43,60}$ organic solvents ${ }^{55,61}$ and in ILs., ${ }^{2,31-33}$ The small-angle scattering profiles of these showed behavior typical of spinodal decomposition: the divergence of scattering intensity correlation length as the critical point is approached. In contrast, in a recent study of PNIPAm in dilute aqueous solution, it was reported that the SANS profiles show a discontinuous change at the critical point where the steepness of the change decreased with the increasing fraction of the meso-diad in the PNIPAm chain, i.e., decreasing cooperativity of hydration..$^{62-64}$ Here, cooperative solvation means that the desorption of a solvent molecule from a site induces the desorption of another solvent molecule from its neighboring site. From this, we concluded that the sharpness of the phase separation results from the cooperative dehydration of PNIPAm at the critical point.

It should be noted that the present study shows a discontinuous change in the scattering from that of dispersed polymer chains to that of large aggregates in dilute solution. Based on the previous study on the PNIPAm dilute aqueous solution, we conjecture that the discontinuous change in SANS profiles observed for PPhEtMA in $d_{8}-\left[\mathrm{C}_{2} \mathrm{mIm}^{+}\right]\left[\mathrm{TFSA}^{-}\right]$also originates from the cooperative dissociation of IL ions at $T_{\mathrm{c}}$.

Earlier in this section, we demonstrated that the phase separation behavior of PPhEtMA in the $\left[\mathrm{C}_{2} \mathrm{mIm}^{+}\right]\left[\mathrm{TFSA}^{-}\right]$system in SANS was well described by the typical functions for the Gaussian chains in dilute solution with a phase separated structure. To investigate the system more quantitatively, we evaluated the thermodynamic parameters as determined from the Zimm plot. At the limit of low- $c$ and low- $q$, a scattering intensity from a polymer solution $I(q)$ can be approximated as follows:

$$
\frac{K c}{I(q)}=M_{\mathrm{w}}{ }^{-1}\left(1+\frac{R_{\mathrm{g}, 0}{ }^{2}}{3} q^{2}\right)+2 A_{2} c
$$

where $K=\Delta \rho^{2} / N_{\mathrm{A}} d_{\text {polymer }}^{2}$ (for the present case, $K=0.0015 \mathrm{~mol} \mathrm{~cm} \mathrm{~g}^{-2}$ ) is the contrast factor. Here, $\Delta \rho$ is the difference of scattering length densities between PPhEtMA and $d_{8}-\left[\mathrm{C}_{2} \mathrm{mIm}^{+}\right]\left[\mathrm{TFSA}^{-}\right]\left(\right.$i.e., $3.044 \times 10^{10} \mathrm{~cm}^{-2}$ ) and $d_{\text {polymer }}, N_{\mathrm{A}}$, $R_{\mathrm{g}, 0}$ and $A_{2}$ are the mass density of PPhEtMA, the Avogadro number, the radius of gyration as $c \rightarrow 0$, and the second virial coefficient, respectively. We used the mass density of the PhEtMA monomer (see Table S1, ESI $\dagger$ ) instead of the corresponding value of PPhEtMA in the analysis, because the mass density of PPhEtMA in solution is unknown.

Fig. 6 shows the Zimm plot at various temperatures $(288,293$, 298, and $303 \mathrm{~K}$ ) below $T_{\mathrm{c}}$. We successfully obtained reasonable values of $M_{\mathrm{w}}$ for PPhEtMA (the obtained $M_{\mathrm{w}}$ values are in the range of $32-34 \mathrm{kDa}$ ) by fitting the data to the function described in eqn (5), confirming the validity of the experiment and the analysis. Fig. 7 shows the temperature dependence of (a) $A_{2}$ and (b) $R_{\mathrm{g}, 0}$ obtained from the Zimm plot. As Fig. 7(a) illustrates,
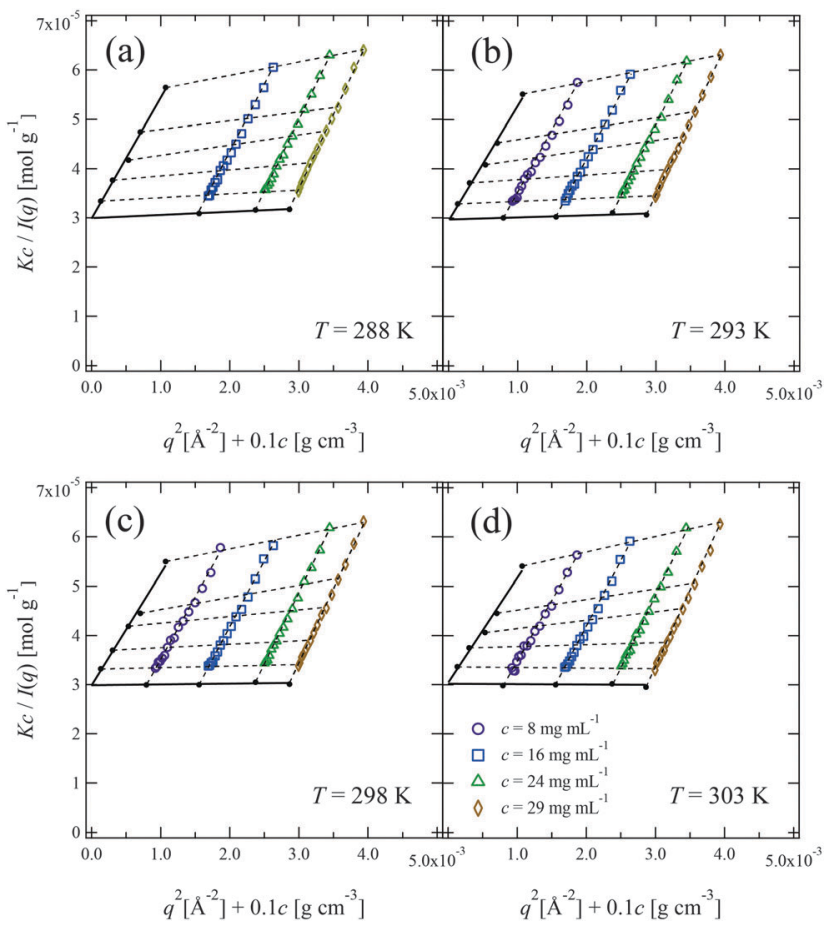

Fig. 6 Zimm plots for the PPhEtMA in $d_{8}-\left[\mathrm{C}_{2} \mathrm{mlm}^{+}\right]\left[\mathrm{TFSA}^{-}\right]$solutions at (a) $T=288 \mathrm{~K}$, (b) $T=293 \mathrm{~K}$, (c) $T=298 \mathrm{~K}$ and (d) $T=303 \mathrm{~K}$.
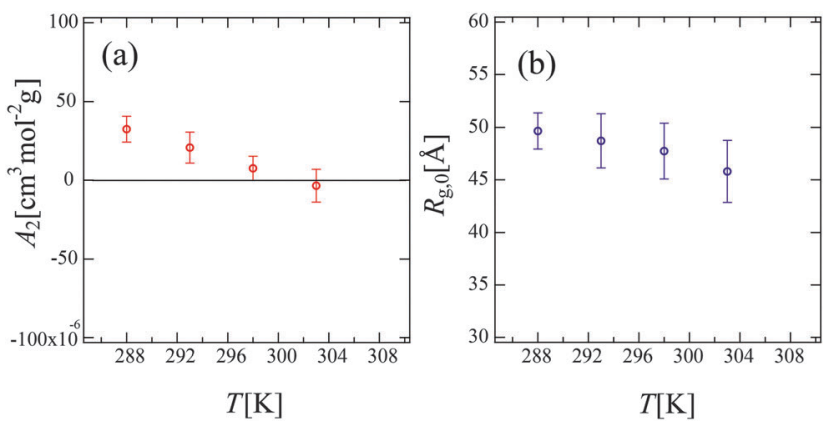

Fig. 7 The temperature dependence of (a) the second virial coefficient, $A_{2}$ and (b) the radius of gyration at $c \rightarrow 0, R_{\mathrm{g}, 0}$ obtained from the Zimm plots shown in Fig. 6.

$A_{2}$ decreased with increasing temperature and then changed its sign at $T=301 \mathrm{~K}$, i.e., the $\Theta$ temperature. This result indicates that the compatibility of PPhEtMA decreases gradually with increasing temperature. As Fig. 7(b) demonstrates, $R_{g, 0}$ was independent of temperature within its error, similar to the results of the DLS experiments (see Fig. 4). The stability of $R_{\mathrm{h}}$ and $R_{\mathrm{g}, 0}$ even near the critical point indicates that the desolvation of IL ions from the solvated PPhEtMA occurs suddenly at the critical temperature. This interpretation is supported by the sudden and discontinuous changes observed in SANS profiles at $T_{\mathrm{c}}$ (see Fig. 5).

\section{Comparison between PPhEtMA and PBnMA in $\left[\mathrm{C}_{2} \mathrm{mIm}^{+}\right]\left[\mathrm{TFSA}^{-}\right]$solutions}

In the former sections, we evaluated the structural parameters for PPhEtMA in the $\left[\mathrm{C}_{2} \mathrm{mIm}^{+}\right]\left[\mathrm{TFSA}^{-}\right]$system. Here, we compare 
the structural parameters of PPhEtMA with those of PBnMA in $\left[\mathrm{C}_{2} \mathrm{mIm}^{+}\right]\left[\mathrm{TFSA}^{-}\right]$to probe the physical origin of the difference between the measured $T_{\mathrm{c}}$ 's $\left(T_{\mathrm{c}}\right.$ of PPhEtMA in $\left[\mathrm{C}_{2} \mathrm{mIm}^{+}\right]\left[\mathrm{TFSA}^{-}\right]$ is approximately $60 \mathrm{~K}$ lower than that of PBnMA). ${ }^{1}$ We have previously reported a SANS study on PBnMA in the $\left[\mathrm{C}_{2} \mathrm{mIm}^{+}\right]\left[\mathrm{TFSA}^{-}\right]$ system in which we estimated the effective interaction parameter $\chi_{\text {eff }}$ in the dilute region where the chain overlapping concentration was also determined by viscosity measurements (Fig. S1, ESI $\dagger){ }^{2}$ Fig. 8 shows the comparison of the temperature dependence of $\chi_{\text {eff }}$ obtained for PPhEtMA and PBnMA using the same analytical procedure of fitting the curve using the function given in eqn (3). The value of $\chi_{\text {eff }}$ thus obtained for PPhEtMA solution at $c=16 \mathrm{mg} \mathrm{mL}^{-1}$ was very close to the value converted from $A_{2}$ obtained using the Zimm plot shown in Fig. S2 (ESI $\dagger$ ), confirming the validity of the curve fitting.

In the vicinity of the critical point, $\chi_{\text {eff }}$ can be approximated as a function of temperature as follows:

$$
\chi_{\mathrm{eff}}=A+\frac{B}{T}
$$

where $A$ and $B$ are the entropic and enthalpic contributions to $\chi_{\text {eff }}$, respectively. ${ }^{33,65}$ As can be seen, the value of $|B|$ obtained for PPhEtMA $\left(=56 \mathrm{~K}^{-1}\right)$ was smaller than the corresponding value obtained for PBnMA $\left(=84 \mathrm{~K}^{-1}\right)$ whereas there was a small difference in the values of $|A|$ obtained for PPhEtMA $(=0.69)$ and for PBnMA (=0.73).

The observed differences between the obtained $|A|$ and $|B|$ for PPhEtMA and PBnMA imply that the chemical modification of PBnMA by the insertion of a methylene group into the side chains lowers the absolute value of the enthalpy of mixing, resulting in the decrease of $T_{\mathrm{c}}$. In our previous study, we pointed out that the dominant microscopic interaction between

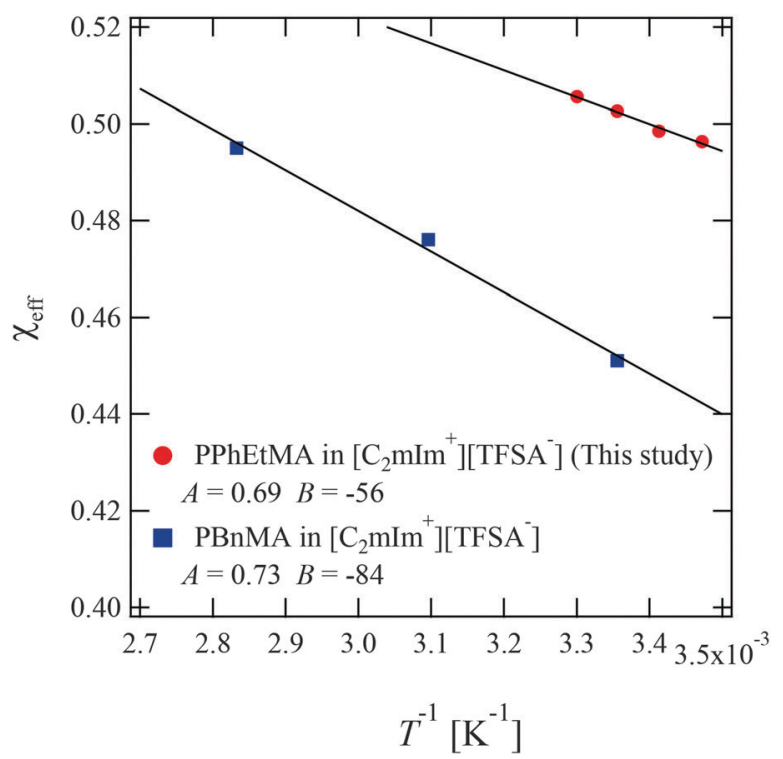

Fig. 8 The temperature dependence of the effective interaction parameter $\chi_{\text {eff }}$ of the PPhEtMA in $\left[\mathrm{C}_{2} \mathrm{mlm}^{+}\right]\left[\mathrm{TFSA}^{-}\right]$solution obtained from the curve fitting shown in Fig. 5, together with the corresponding values for PBnMA in the $\left[\mathrm{C}_{2} \mathrm{mlm}^{+}\right]\left[\mathrm{TFSA}^{-}\right]$solution system. ${ }^{2}$ The solid lines are linear fits to $\chi_{\text {eff }}=A+B / T$; the fitted values for $A$ and $B$ are shown in the graph.
PBnMA and $\left[\mathrm{C}_{2} \mathrm{mIm}^{+}\right]\left[\mathrm{TFSA}^{-}\right]$is the cation- $\pi$ interaction between $\mathrm{C}_{2}$ mIm and PBnMA whereby $\mathrm{C}_{2}$ mIm cations preferentially solvate above and below the benzyl groups in the PBnMA side chains. ${ }^{28}$ Considering this fact, alkyl groups in side chains of PBnMA or PPhEtMA do not contribute to the interactions between $\mathrm{C}_{2}$ mIm cations and the side chains. Hence, we conclude that the lower $|B|$ value of PPhEtMA than PBnMA originates from an increase of unfavorable interactions between PPhEtMA and the IL caused by the insertion of the methylene group.

Furthermore, we compared the values of $A$ and $B$ between the polymer in IL systems and PNIPAm in aqueous solution systems. The obtained values of $|A|$ and $|B|$ for PPhEtMA and PBnMA in $\left[\mathrm{C}_{2} \mathrm{mIm}^{+}\right]\left[\mathrm{TFSA}^{-}\right]$were smaller than those estimated for PNIPAm in aqueous solution using previously reported data $\left(|A|=1.15,|B|=203 \mathrm{~K}^{-1}\right) .{ }^{64}$ This reduced temperature dependence of $\chi_{\text {eff }}$ in IL systems indicates that the phase equilibrium of the IL system can be sensitively affected by a slight change in $A$ or $B$, i.e., chemical modification to the polymer or IL. Hence, it was determined that the strong dependence of $T_{\mathrm{c}}$ on the chemical structure in IL systems originates from the relatively smaller temperature dependence of $\chi_{\text {eff }}$ in these systems.

\section{Conclusions}

The solvated structure and phase behavior of the thermoresponsive polymer PPhEtMA in the $\left[\mathrm{C}_{2} \mathrm{mIm}^{+}\right]\left[\mathrm{TFSA}^{-}\right]$solution system were investigated quantitatively by SANS experiments. The following facts were disclosed: (1) the obtained SANS profiles showed a discontinuous change from that of dispersed polymer chains to that of large aggregates for PPhEtMA in the IL solution, indicating that the phase separation occurs discontinuously at $T_{\mathrm{c}}$. The scattering profiles were analyzed by curve fitting with a single-contact Debye function and by the Zimm plot. The second virial coefficient $A_{2}$ was successfully evaluated, and the results showed that the compatibility of PPhEtMA with the IL gradually decreases with increasing temperature. (2) The absolute value of the enthalpic term $|B|$ in the effective interaction parameter $\chi_{\text {eff }}$ obtained for PPhEtMA was smaller than that for poly(benzyl methacrylate) (PBnMA). This difference in the enthalpic term can be attributed to an increase of the unfavorable interaction between IL and PPhEtMA caused by the insertion of the methylene group. (3) The obtained $|A|$ and $|B|$ values of the polymer in IL systems were significantly smaller than those of the PNIPAm in aqueous solution systems. It was determined that the strong dependence of $T_{\mathrm{c}}$ on the chemical structure in IL systems originated from the relatively-smaller temperature dependence of $\chi_{\text {eff. }}$.

\section{Acknowledgements}

This work has been financially supported by Grants-in-Aid for Scientific Research from the Ministry of Education, Culture, Sports, Science, and Technology (No. 22245018 to M. S.). The experiment (IPTS12408.1) using the General-Purpose SANS (CG-2) at Oak Ridge National Laboratory was supported by the 
US-Japan Cooperative Program on Neutron Scattering. Travel expenses for the experiment were also supported by General User Program for Neutron Scattering Experiments, Institute for Solid State Physics, The University of Tokyo (proposal no. 14906), at JRR-3, Japan Atomic Energy Agency, Tokai, Japan. The High Flux Isotope Reactor and beamline CG2 of ORNL was sponsored by the Scientific User Facilities Division, Office of Basic Energy Sciences, U.S. Department of Energy. K. H. was supported by Japan Society for the Promotion of Science through Program for Leading Graduate Schools (MERIT).

\section{Notes and references}

1 K. Kodama, H. Nanashima, T. Ueki, H. Kokubo and M. Watanabe, Langmuir, 2009, 25, 3820.

2 K. Fujii, T. Ueki, K. Niitsuma, T. Matsunaga, M. Watanabe and M. Shibayama, Polymer, 2011, 52, 1589-1595.

3 T. Welton, Chem. Rev., 1999, 99, 2071-2083.

4 S. Seki, Y. Kobayashi, H. Miyashiro, Y. Ohno, A. Usami, Y. Mita, N. Kihira, M. Watanabe and S. Terada, J. Phys. Chem. B, 2006, 110, 10228-10230.

5 K. Fujii, H. Hamano, H. Doi, X. Song, S. Tsuzuki, H. Hayamizu, S. Seki, Y. Kameda, K. Dokko, M. Watanabe and Y. Umebayashi, J. Phys. Chem. C, 2013, 117, 19314-19324.

6 J. G. Huddleston, H. D. Willauer, R. P. Swatloski, A. E. Visser and R. D. Rogers, Chem. Commun., 1998, 1765-1766.

7 C. Chiappe and D. Pieraccini, J. Phys. Org. Chem., 2005, 18, 275-297.

8 R. Madeira Lau, F. Van Rantwijk, K. R. Seddon and R. A. Sheldon, Org. Lett., 2000, 2, 4189-4191.

9 R. P. Swatloski, S. K. Spear, J. D. Holbrey and R. D. Rogers, J. Am. Chem. Soc., 2002, 124, 4974-4975.

10 F. Rantwijk and R. A. Sheldon, Chem. Rev., 2007, 107, 2757-2785.

11 M. Moniruzzaman, N. Kamiya, K. Nakashima and M. Goto, Green Chem., 2008, 10, 497-500.

12 R. T. Carlin and J. Fuller, Chem. Commun., 1997, 1345.

13 P. Scovazzo, J. Kieft, D. A. Finan, C. Koval, D. DuBois and R. Noble, J. Membr. Sci., 2004, 238, 57-63.

14 M. Galinski, A. Lewandowski and I. Stepniak, Electrochim. Acta, 2006, 51, 5567-5580.

15 N. Winterton, J. Mater. Chem., 2006, 16, 4281.

16 J. M. Lu, F. Yan and J. Texter, Prog. Polym. Sci., 2009, 34, 431-448.

17 T. Ueki and M. Watanabe, Bull. Chem. Soc. Jpn., 2012, 85, 35-50.

18 T. Ueki and M. Watanabe, Chem. Lett., 2006, 35, 964-965.

19 T. Ueki and M. Watanabe, Langmuir, 2007, 23, 988-990.

20 R. Tsuda, K. Kodama, T. Ueki, H. Kokubo, S. Imabayashi and M. Watanabe, Chem. Commun., 2008, 4939.

21 S. Tamura, T. Ueki, K. Ueno, K. Kodama and M. Watanabe, Macromolecules, 2009, 42, 1315.

22 T. Ueki, A. Yamaguchi, N. Ito, K. Kodama, J. Sakamoto, K. Ueno, H. Kokubo and M. Watanabe, Langmuir, 2009, 25, 8845 .
23 T. Ueki, M. Watanabe and T. P. Lodge, Macromolecules, 2009, 42, 1315-1320.

24 H.-N. Lee and T. P. Lodge, J. Phys. Chem. Lett., 2010, 1, 1962-1966.

25 H. N. Lee and T. P. Lodge, J. Phys. Chem. B, 2011, 115, 1971-1977.

26 S. Imaizumi, H. Kokubo and M. Watanabe, Macromolecules, 2012, 45, 401-409.

27 Y. Kitazawa, T. Ueki, K. Niitsuma, S. Imaizumi, T. P. Lodge and M. Watanabe, Soft Matter, 2012, 8, 8067-8074.

28 M. Matsugami, K. Fujii, T. Ueki, Y. Kitazawa, Y. Umebayashi, M. Watanabe and M. Shibayama, Anal. Sci., 2013, 29, 311-314.

29 T. Ueki, A. A. Arai, K. Kodama, S. Kaino, N. Takada, T. Morita, K. Nishikawa and M. Watanabe, Pure Appl. Chem., 2009, 81, 1829-1841.

30 W. Li and P. Wu, Soft Matter, 2013, 9, 11585-11597.

31 H. N. Lee, N. Newell, Z. Bai and T. P. Lodge, Macromolecules, 2012, 45, 3627-3633.

32 H. Asai, K. Fujii, T. Ueki, S. Sawamura, Y. Nakamura, Y. Kitazawa, M. Watanabe, Y.-S. Han, T.-H. Kim and M. Shibayama, Macromolecules, 2013, 46, 1101-1106.

33 M. L. Hoarfrost, Y. He and T. P. Lodge, Macromolecules, 2013, 46, 9464-9472.

34 R. P. White and J. E. G. Lipson, Macromolecules, 2013, 46, 5714-5723.

35 Y. Kitazawa, T. Ueki, S. Imaizumi, T. P. Lodge and M. Watanabe, Chem. Lett., 2014, 43, 204-206.

36 K. Fujii, R. Kanzaki, T. Takamuku, Y. Kameda, S. Kohara, M. Kanakubo, M. Shibayama, S. Ishiguro and Y. Umebayashi, J. Chem. Phys., 2011, 135, 244502.

37 K. Fujii, H. Asai, T. Ueki, T. Sakai, S. Imaizumi, U. Chung, M. Watanabe and M. Shibayama, Soft Matter, 2012, 8, 1756-1759.

38 P. Snedden, A. I. Cooper, K. Scott and N. Winterton, Macromolecules, 2003, 36, 4549-4556.

39 Y. He, Z. Li, P. Simone and T. P. Lodge, J. Am. Chem. Soc., 2006, 128, 2745-2750.

40 S. W. Provencher, Comput. Phys. Commun., 1982, 27, 213-227.

41 H. Tokuda, K. Hayamizu, K. Ishii, M. A. B. H. Susan and M. Watanabe, J. Phys. Chem. B, 2005, 109, 6103.

42 T. Ueki, T. Karino, Y. Kobayashi, M. Shibayama and M. Watanabe, J. Phys. Chem. B, 2007, 111, 4750-4754.

43 M. Shibayama, T. Tanaka and C. C. Han, J. Chem. Phys., 1992, 97, 6829-6841.

44 H. Shirota and K. Horie, Chem. Phys., 1999, 242, 115.

45 H. Shirota, N. Kuwabara, K. Ohkawa and K. Horie, J. Phys. Chem. B, 1999, 103, 10400.

46 C. Strazielle and H. Benoit, Macromolecules, 1975, 8, 203-205. 47 H. Yang, M. Shibayama, R. S. Stein, N. Shimizu and T. Hashimoto, Macromolecules, 1986, 19, 1667-1674.

48 E. L. Atkin, L. A. Kleintjens, R. Koningsveld and L. J. Fetters, Makromol. Chem., 1984, 185, 377-387.

49 M. Shibayama, S. Mizutani and S. Nomura, Macromolecules, 1996, 29, 2019-2024.

50 H. Fujita, Polymer Solution, Elsevier, Amsterdam, 1990. 
51 H. Asai, K. Fujii, T. Ueki, T. Sakai, U. Chung, M. Watanabe, Y. S. Han, T. H. Kim and M. Shibayama, Macromolecules, 2012, 45, 3902-3909.

52 H. Benoit and M. Benmouna, Polymer, 1984, 25, 1059-1067.

53 H. Benoît, C. Picot and M. Benmouna, J. Polym. Sci., Part B: Polym. Phys., 1984, 22, 1545-1548.

54 J. S. Higgins and H. C. Benoit, Polymers and Neutron Scattering, Clarendon Press, Oxford, 1994.

55 Y. Xie, K. F. Ludwig Jr, R. Bansil, P. D. Gallagher, X. Cao and G. Morales, Physica A, 1996, 232, 94-108.

56 B. Hammouda, D. Ho and S. Kline, Macrmolecules, 2002, 35, 8578-8585.

57 Q. Liao, A. V. Dobrynin and M. Rubinstein, Macromolecules, 2006, 39, 1920-1938.

58 M. N. Spiteri, C. E. Williams and F. Boué, Macromolecules, 2007, 40, 6679-6691.
59 A. K. Gupta, J. P. Cotton, E. Marchal, W. Burchard and H. Benoit, Polymer, 1976, 17, 363-366.

60 I. R. Nasimova, T. Karino, S. Okabe, M. Nagao and M. Shibayama, Macromolecules, 2004, 37, 8721-8729.

61 Y. B. Melnichenko, G. D. Wignall, A. W. Van Hook, J. Szydlowski, H. Wilczura and L. P. Rebelo, Macromolecules, 1998, 31, 8436-8438.

62 B. H. Zimm and J. K. Bragg, J. Chem. Phys., 1959, 31, 526-535.

63 Y. Okada and F. Tanaka, Macrmolecules, 2005, 38, 4465-4471.

64 K. Nishi, T. Hiroi, K. Hashimoto, K. Fujii, Y.-S. Hang, T.-H. zKim, Y. Katsumoto and M. Shibayama, Macromolecules, 2013, 46, 6225-6232.

65 A. R. Shultz and P. J. Flory, J. Am. Chem. Soc., 1952, 74, 4760-4767. 